\title{
Five per Second
}

National Cancer Institute

\section{Source}

National Cancer Institute. Five per Second. NCI Thesaurus. Code C67269.

A unit denoting a number of events equal to 5 or quantity equal to 5 expressed in any units per unit of time equal to one second. 